\title{
«Quick, convenient, and clean»: Advancing education in chemistry and nanocatalysis using sol-gel catalysts under flow
}

\author{
Antonino Scurria, ${ }^{1}$ Mario Pagliaro, ${ }^{1}$ Rosaria Ciriminna ${ }^{1}(\varangle)$ \\ ${ }^{I}$ Istituto per lo Studio dei Materiali Nanostrutturati, CNR, via U. La Malfa 153, 90146 Palermo, Italy
}

\begin{abstract}
Removing one key barrier to the industrial uptake of green chemistry and nanocatalysis in the fine and specialty chemical industry requires to fill an ongoing "talent shortage" via expanded chemistry education. In this study we show how the use of hybrid sol-gel catalysts to synthesize fine chemicals and active pharmaceutical ingredients in flow chemistry reactors illustrates new ideas to reshape chemistry education based on recent research outcomes, visualization and digital tools. Several lessons learned from the industrial and academic utilization of these materials in continuous-flow conversions conclude the study.
\end{abstract}

\section{KEYWORDS}

Heterogeneous catalysis; green chemistry; organosilica; flow chemistry; sol-gel; nanocatalysis

\section{Introduction}

Driven by the increasing demand from the pharmaceutical, food and beverage, flavor and fragrance, cosmetic, personal care, electronic and advanced materials industries, in the last thirty years the fine chemical industry has dramatically grown and significantly changed, with productions now chiefly taking place in China and in India [1]. Revenues have gone from \$42 billion in 1993 (with the pharmaceutical customers absorbing $50 \%$ of production) [2], to $\$ 128$ billion in 2014 (with companies making medicines now absorbing $70 \%$ of production) [3].

In a study focusing on the early applications of green chemistry in the fine chemical industry, in 2013 we wrote that "within the next two decades, the fine industry would have relied "on heterogeneously catalyzed processes using molecular, metal and enzymatic catalysts with little or no waste generation, and minimal energy and resource utilization. Reactions will be carried out in flow reactors located in industrial plants 20 or 30 times smaller than today's batch plants of similar output"[4]; adding that industry will accept to undertake the transition to green (i.e., low or zero-waste) processes when their economic advantages in terms of quick payback time, better product quality, and shorter time to market will be made evident.

The study was concluded emphasizing that the industrial uptake of new generation catalysis technology and productions under flow requires to fill a "talent shortage" identified as one of the key barriers to green chemistry the fine and specialty chemical industry $[5,6]$.

Drawing on independent academic and industrial researches using commercial hybrid sol-gel catalysts in continuous processes, in this study we show how the use of these catalysts to synthesize fine chemicals and active pharmaceutical ingredients practically illustrates new ideas to reshape chemistry education based on recent research outcomes, visualization and digital tools [7]. Several lessons learned from the industrial and academic utilization of these materials conclude the study.

\section{Selected Continuous-Flow Conversions}

Comprised of different catalytic species entrapped in an amorphous organosilica matrix, the selected catalysts belong to the commercial SiliaCat (a trademark of SiliCycle) catalyst series. Widely investigated both in batch [8] and continuous [9] processes, these catalysts have been successfully applied to a wide range of processes commonly employed by the fine chemical industry including highly selective olefin reduction and alcohol oxidation, cross-coupling, hydrosilylation, hydrogenolysis, and liquid fat hydrogenation reactions.

Reporting in 2016 the laboratory classroom use of one such catalyst to mediate the full hydrogenation in batch of a wide variety of alkenes and alkynes [10], O'Connor and co-workers were the first to develop green chemistry laboratory experiments based on these new catalytic materials. Since then, even flow chemistry companies started to use these materials to illustrate the advantages of heterogeneous catalysis under flow and show the effectiveness of these materials in streamlining organic synthesis (see below). In the following, we focus on the outcomes of selected independent academic and industrial researches using these catalysts in continuous-flow processes to illustrate concepts in green chemistry and nanocatalysis based on recent research outcomes, in one case visualizing the new synthetic process using digital tools [7].

\subsection{Metal-Free Aerobic Alcohol Oxidation. SiliaCat TEMPO} is an organosilica-immobilized TEMPO (2,2,6,6-tetramethylpiperidine-1-oxyl) catalyst normally employed in the biphasic Anelli-Montanari oxidation of alcohols to carbonyl compounds [11]. In 2013 Hermans and co-workers reported its successful application to the aerobic oxidation of alcohols, using oxygen in combination with a catalytic amount of $\mathrm{HNO}_{3}$ as a $\mathrm{NO}_{x}$ source under continuous three-phase flow conditions [12].

Performed in transparent Teflon tubes in a continuous flow system including a fixed-bed reactor embedding the solid catalyst (h in Scheme 1), a gas, and a liquid reservoir, the reactions used low vaporization enthalpy 1,2-dichloroethane (DCE) as substrate and product solvent to ease solvent recovery via a phase separator. 
The system pressure was set at 5 bar via a back-pressure regulator.

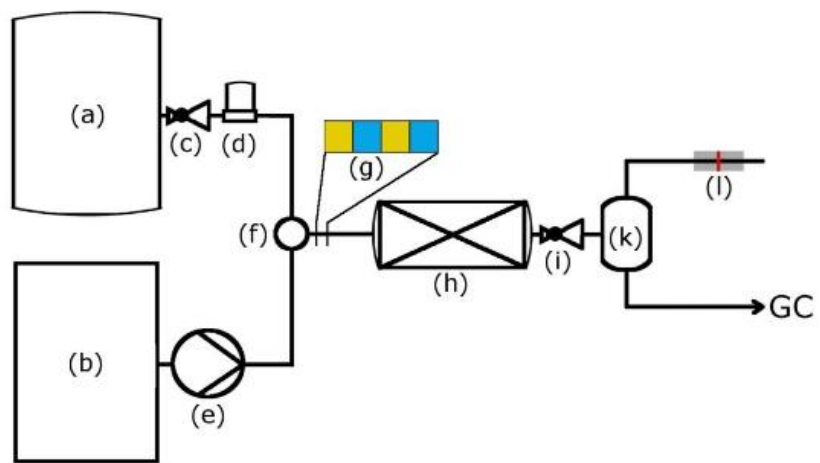

Scheme 1. Experimental set-up: (a) $\mathrm{O}_{2}$ reservoir, (b) liquid reservoir, (c) pressure reduction valve, (d) mass flow controller, (e) HPLC pump, (g) segmented flow, (h) fixed-bed reactor, (i) back-pressure regulator, (k) phase separator and (l) gas-flow passing through transmission IR cell. [Reproduced from Ref.12, with kind permission].

The liquid feed (solvent, alcohol, aqueous $\mathrm{HNO}_{3}$ ) was pumped with an HPLC pump and combined with the $\mathrm{O}_{2}$ gas flow in a T-mixer, generating a gas-liquid slug flow (which efficiently promotes gas-liquid mass transfer [13]). Table 1 shows the excellent performance of the sol-gel hybrid catalyst.

Table 1. Space-time-yields in the aerobic oxidation of benzyl alcohol over different TEMPO-based solid catalysts with $\mathrm{NO}_{\mathrm{x}}$ as co-catalyst. The reaction over SiliaCat TEMPO is under continuous-flow [Reproduced from Ref.12, with kind permission].

\begin{tabular}{|l|l|l|l|}
\hline Ref. & Catalyst & $\mathbf{T}\left({ }^{\circ} \mathrm{C}\right)$ & Space-time-yield $^{\text {a }}$ \\
\hline 12 & SiliaCat $\mathrm{TEMPO}^{\mathrm{b}}$ & 55 & $4.1 \times 10^{5}$ \\
\hline 14 & TEMPO@ $\mathrm{SiO}_{2}{ }^{\mathrm{c}}$ & r.t. & $4.4 \times 10^{2}$ \\
\hline 15 & TEMPO@ $\mathrm{Fe}_{2} \mathrm{O}_{3}{ }^{\mathrm{d}}$ & 50 & $0.83 \times 10^{5}$ \\
\hline
\end{tabular}

${ }^{\mathrm{a}} \mathrm{mol} \mathrm{BzOH}$ (mol TEMPO)-1 ${ }^{-1} \mathrm{~h}^{-1}$ ( reactor volume $)^{-1}$; ${ }^{\mathrm{b}}$ solvent: DCE, co-oxidant: $\mathrm{HNO}_{3}$; ${ }^{\mathrm{c}}$ solvent: DCE, co-oxidant: $\mathrm{NO}_{\mathrm{x}}$ adsorbed on silica; ${ }^{\mathrm{d}}$ solvent: $\mathrm{H}_{2} \mathrm{O}$, co-oxidant: tert-butyl-nitrite.

The space-time-yields for the oxidation of benzyl alcohol over SiliaCat TEMPO $\left(0.7 \mathrm{mmol} \mathrm{g}{ }^{-1}\right.$ TEMPO radicals; void fraction of $65 \%$ ) at $55{ }^{\circ} \mathrm{C}$ and 5 bar $\mathrm{O}_{2}$ with $10 \mathrm{~mol} \% \mathrm{HNO}_{3}$ as co-catalyst in the continuous flow system was three orders of magnitude and 50 times higher than, respectively, the space-time-yields observed using TEMPO@ $\mathrm{SiO}_{2}$ [14] and TEMPO@ $\mathrm{Fe}_{2} \mathrm{O}_{3}$ [15] magnetic nanoparticles under batch with different $\mathrm{NO}_{\mathrm{x}}$ sources as co-oxidants.

Beyond the mild reaction conditions, the two key aspects that make practically applicable this flow catalytic conversion in industry are the prolonged catalyst stability (the catalyst remains stable over several hours, Figure 1) and its broad scope.

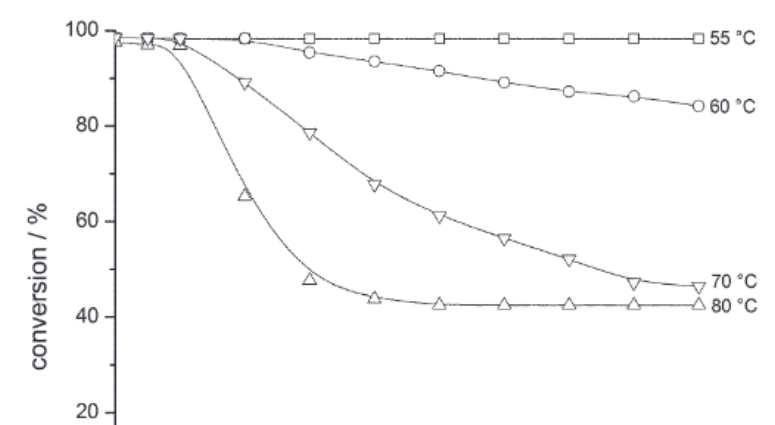

Figure 1. Conversion vs. time-on-stream for different temperatures, a contact time of $0.5 \mathrm{~min}$ in the continuous-flow aerobic oxidation of benzyl mediated by SiliaCat TEMPO under flow in three-phase system (solvent: DCE, $[\mathrm{BzOH}]_{0}=$ $500 \mathrm{mM},\left[\mathrm{HNO}_{3}\right]=5 \mathrm{~mol} \%, 5$ bar $\mathrm{O}_{2}$ ). [Reproduced from Ref.12, with kind permission].

Indeed, several primary and secondary alcohols, including delicate prenol and isoprenol, were quickly converted to aldehydes or ketones with high conversion and selectivity (Table 2).

Table 2. Continuous-flow aerobic oxidation of different alcohols in DCE mediated by $10 \mathrm{~mol} \% \mathrm{HNO}_{3}$ and SiliaCat TEMPO at $55{ }^{\circ} \mathrm{C}$ and 5 bar $\mathrm{O}_{2}$. [Reproduced from Ref.12, with kind permission].

\begin{tabular}{|c|c|c|c|c|}
\hline Substrate & Product & $\mathbf{t}(\min )^{\mathrm{a}}$ & $\begin{array}{l}\text { Conv. } \\
(\%)\end{array}$ & Sel. $(\%)$ \\
\hline & & 0.5 & $98^{\mathrm{b}}$ & $99^{\mathrm{b}}$ \\
\hline $\mathrm{OH}$ & & 2 & 95 & 99 \\
\hline & & 2 & 67 & 88 \\
\hline & & 2 & 93 & 87 \\
\hline & & 2 & 65 & 70 \\
\hline
\end{tabular}

${ }^{\mathrm{a}}$ Contact time not optimized for full conversion; ${ }^{\mathrm{b}} 5 \mathrm{~mol} \% \mathrm{HNO}_{3}$; ${ }^{\mathrm{c}}$ Combined selectivity towards isoprenal and prenal.

The low residence times (up to $2 \mathrm{~min}$ ) is enabled by the excellent mass transfer of the $\mathrm{O}_{2}$ molecules from the gas to the liquid phase, and from the liquid phase to the active sites entrapped within the sol-gel cages. Indeed, the team performed the benzyl alcohol reaction under optimized conditions using catalyst particles of half the size $(0.08-0.12 \mathrm{~mm})$ of the original commercial catalyst (particle size $0.16-0.24 \mathrm{~mm}$ ). No differences in reaction rate or selectivity were observed, showing that intragranular mass-transfer is not the rate limiting step when using SiliaCat TEMPO under flow in the three-phase (gas, liquid, solid) flow reactor.

Herman's team concluded that the use of the catalyst under flow in the newly developed three-phase continuous system "offers a promising alternative to state-of-the-art stoichiometric oxidants or transition metal catalysts for the environmentally benign and economical oxidation of alcohols" [12].

Fine chemical companies generally avoid the use of pure $\mathrm{O}_{2}$ in oxidation reactions in batch reactors because of the high flammability of organic solvents. Continuous flow reactors, in their 
turn, enable the safe and efficient utilization of pure $\mathrm{O}_{2}$ because flow reactors do not feature headspace, inherently preventing the accumulation of potentially combustible oxygen/organic vapor [16], whereas the high reactor surface area/volume ratio eases the quick and efficient dissipation of the heat generated by exothermic oxidation reactions.

2.2 One-Pot Synthesis of Morphine Derivatives. In 2019, Kappe and co-workers in collaboration with researchers from a pharmaceutical company patented new synthetic routes to morphine analogs and derivatives [17]. Widely used in medicine, these compounds function as antagonists and mixed agonist/antagonist. Naltrexone, for example, is administered for the treatment of alcoholism and opioid dependence, and naloxone to reverse opioid overdose.

The new method (Scheme 2) consists in the hydrogenation/oxidative cyclization sequence in which hydrogenation of the starting substrate (14-hydroxymorphinone 1, for example) over $\mathrm{Pd} / \mathrm{C}$ directly is followed by continuous flow oxidation of the resulting solution of oxymorphone $\mathbf{6}$ mediated by SiliaCat DPP-Pd in a packed bed reactor to obtain the desired oxazolidine 7.

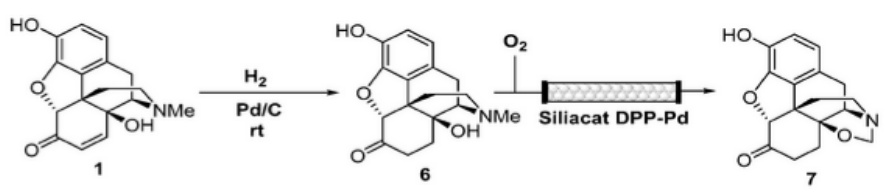

Scheme 2 Semi-continuous hydrogenation/oxidative cyclization synthetic sequence for the preparation of 1,3-oxazolidine. The aerobic oxidation is carried under flow using a packed bed reactor containing SiliaCat DPP-Pd. [Reproduced from Ref.17, with kind permission].

The reaction system used for the synthesis of the opioid antagonists is virtually identical to that used by Hermans and co-workers for alcohol oxidations using SiliaCat TEMPO [12]. A mass flow controller doses the amount of pure $\mathrm{O}_{2}$ retrieved from the reservoir. The liquid solution containing the substrate dissolved in DMA forms a segmented flow prior to enter the fixed-bed column reactor containing the sol-gel entrapped catalyst. A back-pressure regulator regulates the pressure, measured by two pressure sensors one of which is placed directly after the pump and another integrated in the T mixer.

Ethylene glycol was added as co-solvent to dimethylacetamide (DMA) in order to improve the catalyst stability (namely to reactivate the catalyst concurrently during the reaction). The sequential hydrogenation/oxidative cyclization in one pot using heterogeneous Pd catalysts eliminates the need for isolation and handling oxymorphone 6 . The resulting solution is directly subjected to continuous flow oxidation over SiliaCat DPP-Pd. The reaction mixture is collected from the output, with no need of separation of the metal catalyst after the reaction, and directly evaporated under reduced pressure, isolating the oxazolidine 7 with $68 \%$ yield by simple addition of cold water.

Consisting of an organosilica matrix functionalized with diphenylphosphine ligands bound to Pd(II), SiliaCat DPP-Pd has been used for a wide range of Pd-catalyzed cross-coupling reactions [18]. Following a comparative study of the activity and stability of the most common immobilized diarylphosphine- and triarylphosphine-based palladium catalysts employed in C-C coupling reactions (polymer-bound Pd Tetrakis, FiberCat 1001,
EnCat TPP30, and SiliaCat DPP-Pd) in 2015 Kappe and co-workers reported that the sol-gel hybrid catalyst showed far superior leaching resistance and stability with respect to the other catalysts, with very low values of leached palladium [19]. Accordingly, the choice of SiliaCat DPP-Pd for the heterogeneously catalyzed flow oxidation in the new synthesis of morphin analogs, was due to "the high leaching resistance shown by this catalyst for reactions involving heterogeneous $\operatorname{Pd}(0)$ and homogeneous Pd(II) species in the reaction mechanism" [17].

With a relatively large packed bed reactor $(2.4 \mathrm{~mL}$ volume, 760 mg SiliaCat DPP-Pd) to extend the residence time and improve the reaction conversion, high yields and excellent selectivities were obtained. Limited palladium leaching occurred during the oxidation, with the crude solution containing $46.4 \mathrm{ppm} \mathrm{Pd}$, corresponding to $10 \%$ of the total amount of Pd initially contained in the immobilized catalyst. The process, the team concluded, "can be very useful for small scale reactions" [17].

2.3 Chemical Library Synthesis. The usefulness of the SiliaCat palladium catalysts for the quick batch synthesis of a chemical library (a set of related compounds used in high-throughput screening) via different C-C cross-coupling reactions was reported in late 2011 [20]. Quickly run in a parallel, some 76 different Suzuki-Miyaura, Heck-Mizoroki and Sonogashira reactions to prepare biaryls and heterobiaryls (ubiquitous functional molecules synthesized via cross-coupling reactions by the fine chemical and pharmaceutical industries [21]) were demonstrated. No inert or anhydrous conditions were necessary, and all catalysts were found to be reusable with limited leaching of active Pd species in solution (<10 ppm). Only for nitrogen-containing substrates such as pyridines and quinoline leaching levels approaching $30 \mathrm{ppm}$ levels were observed.

Following the first report of practical relevance (using 0.5-1.0 M substrate solutions at flow rate up to $1.0 \mathrm{~mL} \mathrm{~min}^{-1}$ ) of the Suzuki-Miyaura coupling reaction of different aryl halides with phenylboronic acid under flow [22], in 2018 researchers at a pharmaceutical company in Europe demonstrated the efficient application of the SiliaCat DPP-Pd to a compound library synthesis via the Suzuki-Miyaura reaction [23]. Chemical libraries are important in the discovery process of several new functional products, not only bioactive molecules, and flow chemistry using heterogeneous catalysis over the SiliaCat catalysts dramatically reduces the cycle times to get novel functional molecules, for example in the field of organic electronics [24].

The team used a commercial modular flow chemistry system (Vapourtec R-series R4 and R2C+ pumping module, Scheme 4) equipped with an autosampler containing two reagent stock bottles: one filled with the heteroaryl iodide dissolved in DME $(0.05 \mathrm{M})$ and the second with a solution of the aryl boronic acid $(0.08 \mathrm{M})$ and sodium carbonate $(0.15 \mathrm{M})$ in ethanol/water $(3: 2)$. The reagents were delivered via $2 \mathrm{~mL}$ sample injection loops from the autosampler to a $\mathrm{T}$ piece mixer ( $\mathrm{Z}$ in Scheme 4) and then to a standard coiled tube (P1) from which the outflow reaches the (10 $\mathrm{cm} \times 6.6 \mathrm{~mm}$ ) column reactor $(\mathrm{P} 2)$ containing the packed SiliaCat catalyst in powder form. A 8 bar back pressure regulator was connected in-line between the column reactor and the fraction collector.

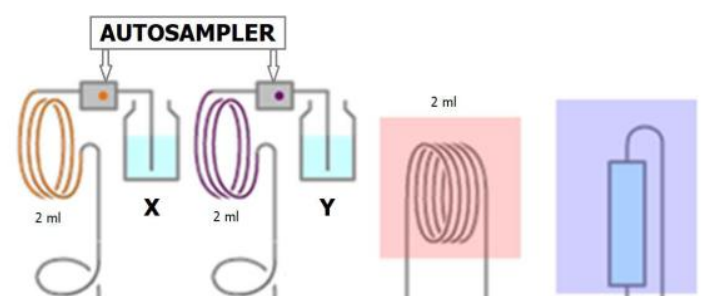


Scheme 4. Flow process set up. [Reproduced from Ref. 23, with kind permission]

After reaction was complete, the reaction mixture collected in 20 $\mathrm{mL}$ vial was evaporated using a centrifugal evaporator. The reaction product was dissolved in a mixture of DCM/water $(2: 1 \mathrm{v} / \mathrm{v})$ and filtered using a phase separation frit. No removal of palladium residues and phosphine ligands was needed. Using this process, 16 new chemical compounds were successfully synthesized using reducing the reaction time from $30 \mathrm{~min}$ in batch to $10 \mathrm{~min}$ in flow, and the reaction temperature from $100{ }^{\circ} \mathrm{C}$ to $90{ }^{\circ} \mathrm{C}$.

2.4 Digital Learning Using Sol-Gel Catalysts under Flow. In late 2017, a company manufacturing flow chemistry reaction systems published online on the World Wide Web a video using SiliaCat DPP-Pd applied to a Suzuki-Miyaura cross-coupling reaction to illustrate the potential of heterogeneous catalysis under flow [25]. In the video, a company's researcher illustrates how using a company' s flow chemistry system (Vapourtec R-Series) it is possible to synthesize 3-phenylpyridine in yield $>80 \%$ (at greater than $95 \%$ purity) with a residence time of just $1 \mathrm{~min}$ and 15 seconds by simply reacting 3-bromopyridine and phenylboronic acid dissolved in organic solvent over the SiliaCat DPP-Pd packed in a glass tubular reactor.

A South Korean company's partner (ChemiSky) republished the video on its website, adding all details of the experiment [26]. A glass column (Omnifit, $6.6 \mathrm{~mm} \times 10 \mathrm{~cm}$ ) is filled with the SiliaCat DDP-Pd catalyst $\left(1.1 \mathrm{~g}, 0.25 \mathrm{mmol} \mathrm{g}{ }^{-1} \mathrm{Pd}\right)$. Reagent bottle A contains a $0.395 \mathrm{M}$ solution of 3-bromopyridine in THF. Reagent bottle B contains a solution $0.19 \mathrm{M}$ phenylboronic acid and $0.23 \mathrm{M}$ $\mathrm{K}_{2} \mathrm{CO}_{3}$ in $\mathrm{H}_{2} \mathrm{O} / \mathrm{EtOH}(2.2: 1, \mathrm{v} / \mathrm{v})$. The system solvent to clean the channels is $\mathrm{H}_{2} \mathrm{O} / \mathrm{EtOH} / \mathrm{THF}(1.14: 1: 1$, v/v).

The column exit is connected to a 8 bar back pressure regulator. This allows the reactor to work above the boiling point of each solvent and enhances the reaction yield. Managed through the Vapourtec Flow Commander software, the system is operated at $100{ }^{\circ} \mathrm{C}$ lowering the residence time to $1 \mathrm{~min}$ and $15 \mathrm{~s}$, setting the flow of both reagent solutions at a molar ratio 1:1.25 (Figure 2), and a constant excess of potassium carbonate. The reaction product is collected in a vial placed under the collection valve of the flow system, instructing the system to collect $2 \mathrm{~g}$ samples.

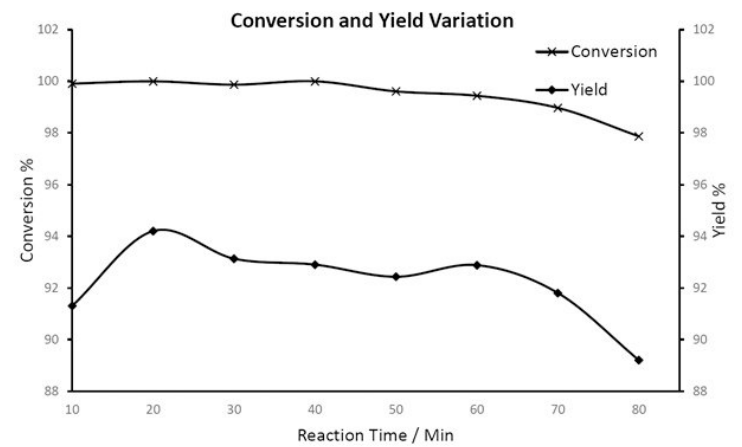

Figure 2. Substrate conversion and yield in in the flow chemistry cross-coupling reaction mediated by SiliaCat DDP-Pd catalyst. [Reproduced from Ref.26, with kind permission]
Reaction was monitored by carrying out an HPLC analysis of product samples every $10 \mathrm{~min}$. The plots in Figure 3 show evidence that yield of 3-phenylpyridine and conversion of 3-bromopyridine remained at high levels during the whole reaction time investigated $(80 \mathrm{~min})$.

\section{Outlook and Conclusions}

En route towards improving education in chemistry via recent research outcomes, visualization and digital tools [7], the story of the use of hybrid sol-gel catalysts of the SiliaCat series in continuous flow processes for the synthesis of valued molecules via different reactions such as aerobic oxidation and cross-coupling reactions teaches several lessons that will be useful to improve the unified teaching and learning of catalysis science and technology [27].

First, as anticipated by Avnir in 1995 [28], the encapsulation of the catalytically active molecular species or metal nanoparticles within the silica sol-gel cages causes the required high catalytic stability for practical applications, due to the physical and chemical stabilization of the catalytic species 3-D entrapped, and not 2-D adsorbed at the outer surface as it happens in surface-heterogenized heterogeneous catalysts such as carbon- and polymer-supported catalysts.

Second, the organic modification of silica with the alkyl groups stabilizes the chemically unstable amorphous mesoporous $\mathrm{SiO}_{2}$, makes it lipohilic and further stabilizes the basic molecular structure of the organosilica polymer now comprised of less strained $(\mathrm{SiO})_{6}$ molecular rings whose interconnection builds the large inner mesoporosity encapsulating and protecting the dopant species [29].

Third, it is the high mesoporosity and huge surface area of the ORMOSIL glasses that, combined with the high mechanical stability of glass, allows direct employment of these hybrid materials of high catalyst load (up to $1 \mathrm{mmol} \mathrm{g}^{-1}$ ) in pressurized flow chemistry reactors by simply adding the powdered catalyst to a packed-bed column reactor.

Fourth, in contrast to what industry did for decades with immobilized homogeneous catalysts due to poor stability of solid catalysts [30], a large number of companies in the fine chemical, pharmaceutical, polymer and organic electronic segments of the chemical industry patented numerous new synthetic processes using these catalysts [31]. This may not be surprising given that reactions mediated by sol-gel organosilica entrapped catalysts often proceed with higher yield and selectivity activity when compared to the corresponding homogeneous catalyst in solution. This has been shown, for example, for SiliaCat TEMPO in place of homogeneous TEMPO in the Anelli-Montanari oxidation of alcohols [32], as well as in that of bacterial cellulose [33], where significantly reduced polymer degradation is observed. Or, in the case of SiliaCat-DDP-Pd, in Stille (when the solid catalyst mediates the conversion with catalyst loadings down to $0.1 \mathrm{~mol} \%$ from $1.0 \mathrm{~mol} \%$ required for homogeneous $\left.\mathrm{Pd}\left(\mathrm{PPh}_{3}\right)_{4}\right)$ or in the heteroarylation (the use of the heterogeneous catalyst in place of $\mathrm{Pd}(\mathrm{OAc})_{2}$ significantly reduces the formation of homo-coupled aryl bromides) reactions [34].

Fifth, being safe and easy to handle (organosilica does not swell in organic solvent even under pressure, does not accumulate static charge, and does not need inert or moisture-free conditions), these 
materials are ideally suited to teach the green chemistry principles via heterogeneous catalysis under flow to undergraduate students [10].

Beyond sol-gel hybrid organosilica catalysts, numerous other newly developed catalytic materials will be applied to the industrial synthesis of fine chemicals via continuous processes mediated by solid catalysts in small flow reactors under mild reaction conditions [35]. For example, a similar hybrid catalyst (poly(dimethyl)silane-supported palladium/alumina) not only is more active than conventional $\mathrm{Pd} / \mathrm{C}$ in the continuous flow hydrogenation of vegetable oils, olefins, squalene, and cholesterol on gram to kilogram scales, but can be used in the production of hydrogenated castor oil on $1.5 \mathrm{~kg} \mathrm{~h}^{-1}$ scale by simply using a large column (10 cm diameter, $1 \mathrm{~m}$ length) remaining stable for more than 1 month [36].

In brief, studying and specializing in nanocatalysis and flow chemistry is and will remain for many years to come a great professional opportunity for chemistry graduates. Fine chemical, personal care, cosmetic and pharmaceutical companies eagerly seek young graduates with proven knowledge and skills in these fields of chemical research. To fill the ongoing knowledge gap, short professional courses aimed to transfer key concepts of flow chemistry to industry's researchers are organized on a routine basis in Europe [37].

In general, after the COVID-19 outbreak, with the immediate need for hydroxychloroquine until then mostly used to prevent malaria and treat the symptoms of lupus and rheumatoid arthritis [38], policy makers in world's developed and developing countries have understood that building or rebuilding the fine chemicals industry is a national strategic need [39]. Flow chemistry and heterogeneous catalysis over molecular, metal, and enzymatic solid catalysts are the key enabling technologies that will allow to build (or to rebuild, in the case of industrially developed countries in Europe, former Soviet countries and North America) the required large and diversified production capacity. Enhanced education in catalysis based on a unified approach using the organic chemistry reaction mechanism expanded by nanochemistry concepts [27] will play a decisive role in supplying industry with the young talented professionals whose lack was still noted in the mid 2010s [5,6].

\section{Authors ORCID}

Antonino Scurria: 0000-0001-5624-6833

Mario Pagliaro: 0000-0002-5096-329X

Rosaria Ciriminna: 0000-0001-6596-1572

\section{Competing interests}

The authors declare no competing financial interest.

\section{Funding Info}

The authors did not receive any funds for this study.

\section{Acknowledgements}

We are indebted to Dr Valerica Pandarus and Dr François Béland, SiliCycle, Canada, and to Professors Laura M. Ilharco and Alexandra Fidalgo, University of Lisboa, Instituto Superior Técnico, for many years of research collaboration on catalytic sol-gel materials. M.P. thanks Professor Vladimir Vinogradov, ITMO University, St. Petersburg, for the invitation to give a lecture for the SCAMT Workshop Week (5-11 July, 2020).

\section{References}

[1] P. Pollak, Fine Chemicals, $2^{\text {nd }}$ edition, Wiley, Hoboken (NJ): 2011.

[2] A. Cybulski, M.M. Sharma, R.A. Sheldon, J.A. Moulijn, Fine Chemicals Manufacture, Elsevier, Amsterdam: 2001.

[3] J. Ramakers, A fine art, Eur. Pharm. Manuf. 2016, 16 (4), 18-19.

[4] R. Ciriminna, M. Pagliaro, Green Chemistry in the Fine Chemicals and Pharmaceutical Industries, Org. Process Res. Devel. 2013, 17, 1479-1484.

[5] M. Pagliaro, Advancing Nanochemistry Education, Chem. Eur. J. 2015, 21, 11931-11936.

[6] T. Jamison cit. In: D. Stanton, Lack of talent will hamper continuous manufacturing adoption, says MIT Prof, In-Pharmatechnologist.com, 13 May 2015. https://www.outsourcing-pharma.com/Article/2015/05/13/Lack-of-talentwill-hamper-continuous-manufacturing-uptake-MIT-Prof\# (last time accessed March 30, 2021).

[7] M. Pagliaro, Chemistry Education Fostering Creativity in the Digital Era, Isr. J. Chem. 2019, 59, 565-571.

[8] R. Ciriminna, V. Pandarus, A. Fidalgo, L. M. Ilharco, F. Béland, M. Pagliaro, SiliaCat: A Versatile Catalyst Series for Synthetic Organic Chemistry, Org. Process Res. Devel. 2015, 19, 755-768.

[9] R. Ciriminna, V. Pandarus, F. Béland, M. Pagliaro, Fine Chemical Synthesis Under Flow Using the SiliaCat Catalysts, Catal. Sci. Technol. 2016, 6, 4678-4685.

[10] C. Pelfrey, S. Ensel, K. O'Connor, SiliaCat Pd(0), a New Green Hydrogenation Catalyst for the Undergraduate Organic Chemistry Laboratory, Chem. Educat. 2016, 21, 40-42.

[11] A. Michaud, G. Gingras, M. Morin, F. Béland, R. Ciriminna, D. Avnir, M. Pagliaro, SiliaCat TEMPO: An effective and useful oxidizing catalyst, Org. Process Res. Dev. 2007, 11, 766-768.

[12] C. Aellig, D. Scholz, S. Conrad, I. Hermans, Intensification of TEMPO-mediated aerobic alcohol oxidations under three-phase flow conditions, Green Chem. 2013, 15, 1975-1980.

[13] R. S. Abiev, I. V. Lavretsov, Hydrodynamics of gas-liquid Taylor flow and liquid-solid mass transfer in mini channels: Theory and experiment, Chem. Eng. J. 2011, 176-177, 57-64.

[14] L. Di, Z. Hua, Porous Silica Beads Supported TEMPO and Adsorbed $\mathrm{NO}_{\mathrm{x}}\left(\mathrm{PSB}-\mathrm{TEMPO} / \mathrm{NO}_{\mathrm{x}}\right)$ : An Efficient Heterogeneous Catalytic System for the Oxidation of Alcohols under Mild Conditions, Adv. Synth. Catal. 2011, 353, 1253-1259.

[15] B. Karimi, E. Farhangi, A Highly Recyclable Magnetic Core-Shell Nanoparticle-Supported TEMPO catalyst for Efficient Metal- and Halogen-Free Aerobic Oxidation of Alcohols in Water, Chem. Eur. J. 2011, 17, 6056-6060.

[16] C. A. Hone, C. O. Kappe, The Use of Molecular Oxygen for Liquid Phase Aerobic Oxidations in Continuous Flow, Top. Curr. Chem. 2019, $377,2$.

[17] C. O. Kappe, B. Gutmann, U. Weigl, P. Egli, D.P. Cox, D. Cantillo, Processes and Oxazolidine-Containing Intermediates for the Preparation of Morphine Analogs and Derivatives, WO2017185004A1, 21 April 2017.

[18] M. Lemay, V. Pandarus, M. Simard, O. Marion, L. Tremblay, F. Béland SiliaCat S-Pd and SiliaCat DPP-Pd: Highly Reactive and Reusable Heterogeneous Silica-Based Palladium Catalysts, Top. Catal. 2010, 53, 1059-1062.

[19] R. Greco, W. Goessler, D. Cantillo, C. O. Kappe, Benchmarking Immobilized Di- and Triarylphosphine Palladium Catalysts for Continuous-Flow Cross-Coupling Reactions: Efficiency, Durability, and Metal Leaching Studies, ACS Catal. 2015, 5, 1303-1312.

[20] V. Pandarus, G. Gingras, F. Béland, R. Ciriminna, M. Pagliaro, Efficient Screening and Library Generation in Parallel C-C Coupling Reactions Mediated by Organosilica SiliaCat Palladium Catalysts, Org. Process Res. Dev. 2012, 16, 117-122.

[21] A. Dumrath, C. Lübbe, M. Beller, Palladium-Catalyzed Cross-Coupling Reactions - Industrial Applications In Palladium-Catalyzed Coupling Reactions, Edited by A. Molnár, Wiley-VCH, Weinheim: 2013; pp. 
445-489.

[22] V. Pandarus, G. Gingras, F. Béland, R. Ciriminna, M. Pagliaro, Process Intensification of the Suzuki-Miyaura Reaction over Sol-Gel Entrapped Catalyst SiliaCat DPP-Pd Under Conditions of Continuous Flow, Org. Process Res. Dev. 2014, 18, 1550-1555.

[23] R. Zadravec, I. Vujasinovic, Suzuki Coupling in Continuous Flow: Efficient Application in Library Synthesis, $38^{\text {th }}$ European School of Medicinal Chemistry ESMEC, Urbino, Italy, July 1-5, 2018. https://fidelta.eu/cms/wp-content/uploads/2020/07/Poster_2018_Zadrave c_Vujasinovic_Urbino_Suzuki-coupling-in-continuous-flow.pdf (last time accessed March 30, 2021).

[24] A.-J. Payne, A. D. Hendsbee, S. M. McAfee, D. K. Paul, K. Karan, G. C. Welch, Synthesis and structure-property relationships of phthalimide and naphthalimide based organic $\pi$-conjugated small molecules, Phys. Chem. Chem. Phys. 2016, 18, 14709-14719.

[25] Vapourtec, Vapourtec Flow Chemistry Suzuki Coupling, YouTube, 1 November 2017. https://www.youtube.com/watch?v=Y_HYlw9iIE (last time accessed March 30, 2021).

[26] ChemiSky, Flow Chemistry: Suzuki Coupling Case Study, Naver, 16 April 2020. https://post.naver.com/viewer/postView.nhn?volumeNo=28004188\&me mberNo $=856950$ (last time accessed March 30, 2021).

[27] M. Pagliaro, «Catalysis: A unified approach»: A new course in catalysis science and technology, J. Flow Chem. 2021, 11, 53-58.

[28] D. Avnir, Organic Chemistry within Ceramic Matrixes: Doped Sol-Gel Materials, Acc. Chem. Res. 1995, 28, 328-334.

[29] R. Ciriminna, L. M. Ilharco, V. Pandarus, A. Fidalgo, F. Béland, M. Pagliaro, Towards waste free organic synthesis using nanostructured hybrid silicas, Nanoscale 2014, 6, 6293-6300.

[30] S. Hübner, J. G. de Vries, V. Farina, Why Does Industry Not Use Immobilized Transition Metal Complexes as Catalysts?, Adv. Synth. Catal. 2016, 358, 3-25.

[31] V. Pandarus, R. Ciriminna, F. Béland, M. Pagliaro, Making fine chemicals, nanomaterials and pharmaceutical ingredients over over SiliaCat catalysts, Appl. Mater. Today 2020, 20, 100661.

[32] R. Ciriminna, C. Bolm, T. Fey, M. Pagliaro, Sol-Gel Ormosils Doped with TEMPO as Recyclable Catalysts for the Selective Oxidation of Alcohols, Adv. Synth. Catal. 2002, 344, 159-163.

[33] S.-H. Jun, S.-G. Park, N.-G. Kang, One-Pot Method of Synthesizing TEMPO-Oxidized Bacterial Cellulose Nanofibers Using Immobilized TEMPO for Skincare Applications, Polymers 2019, 11, 1044.

[34] S. M. McAfee, J. S. J. McCahill, C. M. Macaulay, A. D. Hendsbee, G. C. Welch, Utility of a heterogeneous palladium catalyst for the synthesis of a molecular semiconductor via Stille, Suzuki, and direct heteroarylation cross-coupling reactions, RSC Adv. 2015, 5, 26097-26106.

[35] R. Ciriminna, M. Pagliaro, R. Luque, Heterogeneous catalysis under flow for the $21^{\text {st }}$ century fine chemical industry, Green Energy Environ. 2021, DOI: 10.1016/j.gee.2020.09.013

[36] S. Kobayashi, M. Okumura, Y. Akatsuka, H. Miyamura, M. Ueno, H. Oyamada, Powerful Continuous-Flow Hydrogenation by using Poly(dimethyl)silane-Supported Palladium Catalysts, ChemCatChem 2015, 7, 4025-4029.

[37] See for example, Scientific Update, Flow Chemistry, 3-4 June 2020. https://www.scientificupdate.com/training_courses/flow-chemistry-5/202 00603/ (last time accessed March 30, 2021).

[38] M. Pagliaro, F. Meneguzzo, Hydroxychloroquine for the Treatment of COVID-19: Evidence, Possible Mode of Action and Industrial Supply of the Drug, Medic. Res. 2020, 4, 200014.

[39] R. Ciriminna, I. L. Simakova, M. Pagliaro, D. Yu. Murzin, A scientometric analysis of catalysis research, J. Scientometr. Res. 2020, 9 , 335-343. 
